\title{
Vertical slow drag of an intruder in a laterally confined granular medium
}

\author{
Agnès Duri*, Sandra Mandato, Frédéric Mabille, Bernard Cuq, and Thierry Ruiz \\ UMR IATE 1208 CIRAD/INRA/Montpellier SupAgro/Université Montpellier, 2 Place Pierre Viala, 34060 Montpellier Cedex 5, France
}

\begin{abstract}
The study of force transmission and typologies of motions in a powder bed under low shear solicitation constitutes a challenging issue to achieve a monitoring of most of processes. This work relies on the characterisation of particle mobility in the neighbourhood of a moving intruder in ascendant vertical motion. Vertical force measurements and Particle Image Velocimetry (PIV) technique are used to identify different typologies of particle motions (compaction, loading and rupture of the chain forces, lateral collapsing, avalanches...) of a granular bed ensiled in a semi-confined cell and with a free surface on its top. Force fluctuations during the blade displacement are highlighted and correlated with the dimensionless blade speed but also with the force profile in the static state. The velocity field analysis allows quantifying the shear-banding characteristics corresponding to the blade influence on grain displacements and the impact of the intruder in the motion direction. This signal contains periodical fluctuations which are correlated with the vertical force fluctuations in a specific zone of the granular bed.
\end{abstract}

\section{Introduction}

The description of stress and velocity fields induced by an object (or intruder) moving in a granular medium is of great interest in a wide range of industrial applications. For example, mechanisms involved in wet agglomeration are highly dependent on the knowledge of granular flows under the mechanical solicitation induced by the blade of the mixer. The ability of a mixer to agglomerate hydrated particles depends on its aptitude to induce a stress field in the powder bed in order to enhance the contact and adhesion between hydrated particles through capillary bridges. The study of stress transmission and typologies of motions in a powder bed under mechanical solicitation constitutes then a challenging issue to achieve a monitoring of process like agglomeration or mixing.

Experimentally, Particle Image Velocimetry (PIV) gives access to the powder behaviour by the analysis of velocity fields and particle trajectories. Different configurations have been studied: the vertical penetration of an intruder into a granular medium [1-3] or the horizontal motion of an intruder at a fixed depth in the medium, either entirely burried [4-6] or not [7-8]. A relevant result obtained by Albert et al. [7] is that the behaviour of the drag force in the low velocity regime is dominated by the inhomogeneous distribution of the stress in the static granular media.

This work relies on the analysis of the granular flow induced by an intruder in a semi-confined with a freesurface configuration. A rheophysical device and PIV technique are used to qualify the behaviour of granular materials. A cell with glass walls allows identifying particle motions in two dimensions. A rectangular blade is linked to a texture analyzer which allows an adjustable blade speed and the recording of the vertical force. Using a high-speed camera placed in front of the transparent glass of the cell, velocity fields are obtained by a PIV technique conducted during the blade motion. Different values of a characteristic speeds are tested by varying the blade speed from 1 to $40 \mathrm{~mm} . \mathrm{s}^{-1}$.

\section{Materials and methods}

\subsection{Granular material}

Experiments are conducted using durum wheat semolina of industrial quality as granular material. The main characteristics of semolina are: its dry basis water content of $16.8 \pm 0.4 \%$ measured by drying at $105^{\circ} \mathrm{C}$ for $24 \mathrm{~h}$ [9], its median diameter $d_{50}$ of $287 \pm 8 \mu \mathrm{m}$ measured with a laser granulometer (Coulter TMLS 230, Malvern, England) and its true density of $\rho_{s}=1.478 \pm$ $0.005 \mathrm{~g} . \mathrm{cm}^{-3}$ determined with a nitrogen pycnometer (Ultrapycnometer 1000, Quantachrome Instruments).

\subsection{Rheophysical device}

The rheolphysical device (see Fig. 1), developed by Mandato et al. [10], is composed of an open glass cell container (160 mm height, $100 \mathrm{~mm}$ length, $51 \mathrm{~mm}$ width), a rectangular steel blade $(0.97 \mathrm{~mm}$ thickness, $44.8 \mathrm{~mm}$ length, $19.7 \mathrm{~mm}$ width), screwed on a rod linked to a load cell of a texture analyzer (TA.XT plus, Table Micro System), which allows an ascendant vertical

\footnotetext{
Corresponding author: agnes.duri@inra.fr
} 
motion of the blade with an adjustable speed, and a recording of the vertical force in the powder bed.

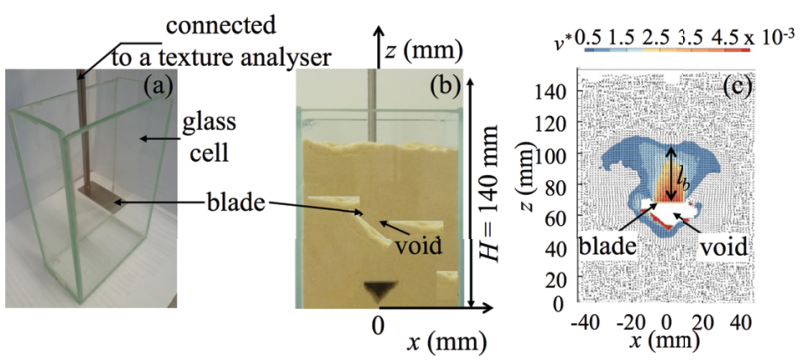

Fig. 1. (a) Picture of the experimental glass cell and the blade, (b) Picture of the granular bed, (c) Instantaneous velocity field image for a specific $z$ position.

Force measurements, $F_{z}$, under dynamic conditions are recorded during the vertical rise of the blade in the cell. Before each test, the blade is first positioned at the bottom of the empty cell. Semolina is sprinkled with a funnel (outlet diameter of $12.3 \mathrm{~mm}$ ) in the middle of the cell until it reaches a height $H$ of $140 \mathrm{~mm}$ which corresponds to a compactness $\phi$ of 0.446 . The blade is moved up for seven different vertical speeds $v_{b}(1,2.5,5$, $10,20,30$, and $40 \mathrm{~mm} \cdot \mathrm{s}^{-1}$ ) with three replications each. The blade speed is rescaled by the characteristic speed of grains in gravity, $v^{*}=v_{b}\left(g d_{50}\right)^{-0.5}(g$ is the acceleration due to gravity). Thus, $v^{*}$ ranges between 0 and 0.75 . The lower $v^{*}$, the longer is the rearrangement time of the grains in the neighbourhood of the moving blade. The experiment at $v^{*}=0$ corresponds to the static vertical force profile of the granular medium in the center $(x=0$ $\mathrm{mm}$ ) of the bed.

The visualization of the semolina grain motion in the neighbourhood of the blade is carried out using a highspeed camera of type CMOS monochrome, with a recording frequency of 100 frames per second and image resolution of $1392 \times 1024$ pixels. In the case of granular flows, because the number of tracers is high, each 8-bit frame is divided into interest areas of $32 \times 32$ pixels, each with an overlap of 60 . Velocity fields are obtained by PIV calculations performed by Direct PIV software (R\&D Vision, France). From them, we have determined the blade vertical influence length, $L_{b}$, which is the vertical height of the zone above the blade, at $x=0 \mathrm{~mm}$ (i.e. in the middle of the cell) where the vertical velocity gradients are localized (Fig. 1c). Values of $L_{b}$ are calculated as a function of the blade height during its vertical rise in the powder bed for each dimensionless speed.

\section{Results and discussion}

First of all, the static profile $\left(v^{*}=0\right)$ is different from the shape of the well-known Janssen profile [11-12]. From the surface, the local weight increases linearly with the depth until a maximum and then decreases until the bottom. In this experiment, as the height of the granular bed is less than the Janssen length $(\sim 260 \mathrm{~mm})$, the level of the local vertical force does not attempt its saturation.
However such a shape, with a decrease of the profile near the bottom, has already been highlighted experimentally by Mandato et al. [10] and numerically by Ovarlez and Clément [13]. This side effect is explained by the deflection of the vertical forces which takes place during the pouring of the cell and is similar with the well-known 'hole heap' [14].

When the blade rises from the bottom to the free surface, disturbances of the local vertical force profile are induced by its motion and the dissipative ability of the network. Transmission of the forces depends on the persistence of the subnetwork of large forces [15] during the blade motion, and this point is linked with the jamming state or the relative mobility of the particles in the bed. The non-uniformity of the initial force distribution in the granular packing and the difference in four different limit conditions between particles and blade, side walls, bottom and free surface, lead to a succession of phenomena. These latter ones can be identified from the measurements of the vertical force during the blade displacement for the different velocities (Fig. 2), and from the velocity fields obtained by PIV (Fig. 1c). With this experimental configuration, lateral shear banding is located in a thin zone at the vicinity of the blade. Considering that the lateral pressure of the shear zone is equal to the static pressure at the same depth and the shear rate approximated by the ratio of the blade speed and the thickness of the shear banding, the inertial number ranges between $10^{-4}$ and $10^{-3}$ [16]. The flowing regime in the sheared band laterally close to the blade is then found to be mostly quasi-static whatever the blade speed.

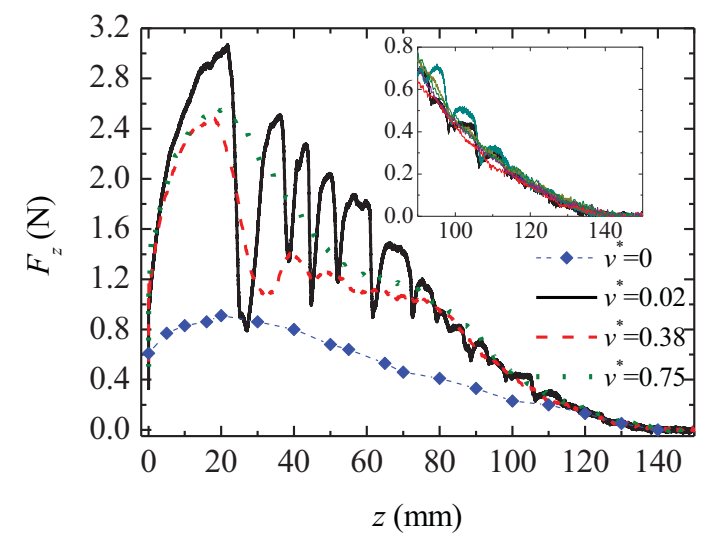

Fig. 2. Examples of $F_{z}$ profiles obtained during ascendant blade displacement for $v^{*}=0,0.02,0.38,0.75$. Inset: $F_{z}$ profiles for all $v^{*}$ in the relaxation phase.

For each solicitation, the shape of the force curves suggests that the dynamics of the granular medium can be subdivided from the bottom to the surface into the three following phases: (i) loading, (ii) fluctuations (loading/rupture), and (iii) final relaxation (Fig. 2).

Loading phase - At the bottom, the initial vertical force corresponds to the static value. During the rise of the blade, a first sharp increase of the force until a first maximum is observed (Fig. 2). At the first peak, the 
maximum vertical force value (mean value $\approx 2.59 \pm 0.26$ $\mathrm{N})$ and its position (mean $=18.68 \pm 2.41 \mathrm{~mm}$ ) are quite uncorrelated with the dimensionless speed. However, the position of this peak corresponds to the position of the highest force of the static profile. During this loading phase, a rearrangement of the inter-granular voids above the blade is observed and corresponds to the creation of a void volume taking place just below the blade (Fig. 1b). The void volume comes from the compaction of the granular layers upon the blade. As the initial compactness of the powder bed $(0.45)$ is lower than the critical compactness above which the granular medium dilates to allow particle rearrangements, it is relevant to assume that a compacted zone is formed just above the blade. During the formation of this compacted zone and all along the blade displacement, particles above the blade are evacuated by micro-avalanches in the free volume and form a concave heap (crater-like) on both sides of the blade. Whatever $v^{*}$, the height of the concave heap depends on the blade width $(1.97 \mathrm{~cm})$ and the angle of repose of semolina $\left(43.8^{\circ}\right)$. The formation of this hole takes place before the force reaches its first peak and corresponds to a small inflexion in the force profile.

Fluctuation phase - After the initial load followed by the break of the force network (decrease of the force just after the first peak), the level of force becomes equal to its static value (first minimum of the force profile), whatever is the blade speed. This result is interpreted as the weak extension of the zone in front of the blade which can be induced by the influence of the first intrusion in the granular media. Then periodic fluctuations are observed (Fig. 2). They correspond to successive phases of loading, compaction, breakage, and relaxation of the force network mobilized by the transmission of the shear stress. These fluctuations are different from the first load, but possess similar characteristics between them. Analysis of the intensities and the frequencies, developed at each cycle, highlights their periodicity which depends on the blade speed.

Loadings are linked with the force intensity of intergranular vaults which act against the blade upward motion and deflect laterally the forces, and to the compaction of the bed zone located just at the top of the blade and up to the front of the disturbance zone vertically generated (Fig. 1c). When the applied force reaches the failure threshold of the force network, particles located in the rupture zones collapse and are then evacuated by avalanches in the void zone under the blade. In the same time, the force exerted on the blade is rapidly relaxed. After this discharge, the measured force increases progressively by the emergence of a new vertical contact network activated by the vertical motion (Fig. 2). This mechanism is periodic until it weakens entirely. Gravish et al. [8] also reported successions of compaction and breakage phenomena on experimental curves of force/displacement relative to a blade-like sensor moving horizontally at a constant speed in a bed of sand or glass beads. However, in this particular case, the constant weight of the displaced granular media ensures a regularity of the frequency.
Relaxation phase - When the blade enters in the highest part of the powder bed, vertical force progressively decreases until to reach the zero value (inset of Fig. 2). This relaxation is independent of the blade speed and can be explained by both a lack of global stiffness of the granular medium and the decrease of the mass weighing on the blade.

Vertical transmission of the force - The blade vertical influence length, $L_{b}$, is defined from the velocity field and represents the distance between the blade at $x=0$ $\mathrm{mm}$ (i.e. in the middle of the cell) and the front of zone where particles are moving (Fig. 1c). Vertical velocity gradients are localized in this zone. Values of $L_{b}$ are determined as a function of the blade height during its vertical rise in the powder bed and for each dimensionless speed (Fig. 3).

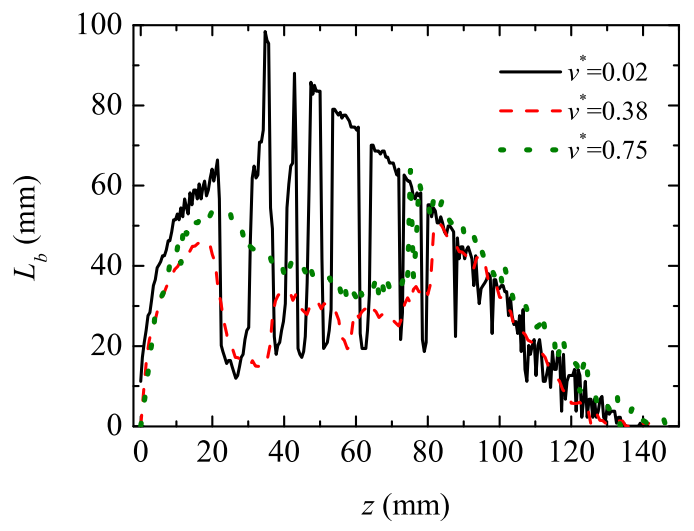

Fig. 3. Examples of $L_{b}$ profiles obtained during ascendant blade displacement for $v^{*}=-0.02,0.38,0.75$.

The vertical extension of the velocity gradient seems to be limited only by phenomenon of vaults rupture, which sets against the propagation of the vertical transmission of force. For each $v^{*}$ and more particularly for $v^{*}<0.2$, the blade vertical influence length fluctuates between two extrema ( $\min$ and $\max$ ). The persistence of a zone of grains in motion above the blade even after the rupture of the force network makes $L_{b}$ always different from zero. A dihedral-shape assembly of grains constitutes a permanent disturbance zone induced by the blade motion. In this coherent zone, the horizontal components of velocity vectors are almost equal to zero and grains are in a jamming state The extension of this zone increases with $v^{*}$. It can be explained by a higher volume of particles dragged by the blade when $v^{*}$ increases. On the contrary, the extension of the fluctuating zone above the permanent one decreases with $v^{*}$. At higher $v^{*}$, the characteristic time required for particle rearrangement is not sufficiently high relatively to the solicitation time. The force networks are alternatively recreated and removed and do not allow enough time to establish loading and rupture cycles of the vaults above the blade. The force transmission from the blade to the surface occurs gradually at the grain 
scale through a vertical contact network established above the blade. Thus, when the network is not persistent in a sufficiently long time comparatively to the characteristic time of solicitation, the transmission is limited in space. On the contrary, the lower is $v^{*}$, the higher is the distance over which the vertical contact network spreads between grains. The extension of the vertical transmission of force is then higher for lower $v^{*}$. Grains of the fluctuating zone are not anymore correlated with the blade which use the benefit of mobility to reach another arrangement.
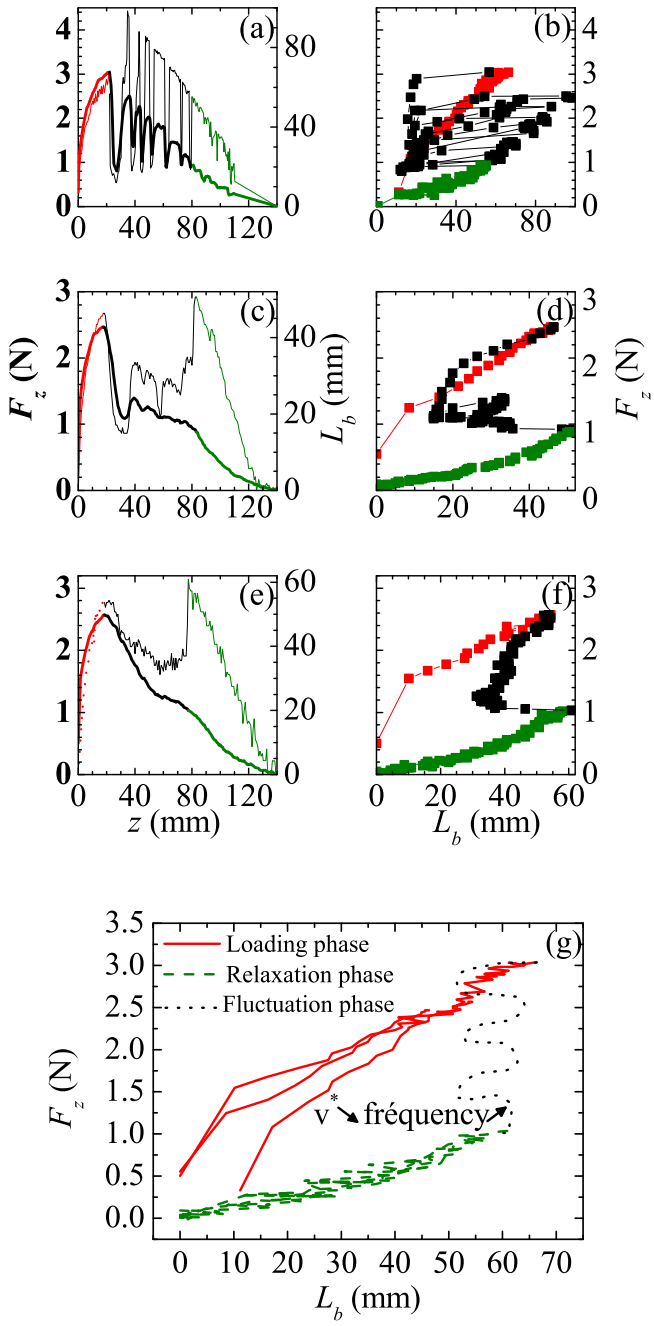

Fig. 4. (in color) $F_{z}$ and $L_{b}$ profiles obtained during ascendant blade displacement for $v^{*}=0.02$ (a), 0.38 (c), 0.75 (e). $F_{z}$ as a function of $L_{b}$ for $v^{*}=0.02$ (b), 0.38 (d), 0.75 (f). Focus on loading and relaxation phases for $v^{*}=0.02,0.38,0.75(\mathrm{~g})$.

The coupled analysis of the dynamic and kinematic curves shows a marked correlation between the vertical force applied on the blade and the length influence of the blade with a small phase shift (Fig. 4). Loading and relaxation phases are independent of $v^{*}$ (Fig. $4 \mathrm{~g}$ ). Fluctuation phase highlights a greater dependence with $v^{*}$, and it is amplified by slow solicitations as described just above.

In any case the force follows the decrease of $L_{b}$, which can be explained by the disjunction from the vertical contact network of a volume of grains which is weighing on the blade until then.

\section{Conclusion}

In this work, a better understanding of the different phenomena occurring in the neighbourhood of an intruder (i.e. flat blade) vertically moving in a laterally confined granular bed with a free-surface at its top is achieved. The PIV analysis allows identifying different types of particles motions (compaction, loading and rupture of the chain forces, lateral collapsing, avalanches, etc.). A detailed comparison between the length of the velocity gradient vertically generated above the blade $\left(L_{b}\right)$ and the vertical force $\left(F_{z}\right)$ perceived by the blade during its rise is carried out. It highlights periodical fluctuations of $L_{b}$ which are correlated with $F_{z}$ fluctuations in a specific zone between the bottom and a characteristic height. Such results contribute to give relevant data which will help in the understanding of granular mobility in a low shear mixer.

\section{Acknowledgements}

This research was financially supported by AgroFlow ANS project from CEPIA department of INRA.

\section{References}

1. Y.C. Zhou, A.B. Yu, R.L. Stewart, J. Bridgwater, Chem. Eng. Sci. 59, 1343-1364 (2004)

2. F. Pacheco-Vázquez, J.C. Ruiz-Suárez, Nature Communications 1, 123 (2010)

3. A. Seguin, Y. Bertho, P. Gondret, J. Crassous, Phys. Rev. Lett. 107, 048001 (2011)

4. D. Chehata, R. Zenit, C.R. Wassgren, Phys. Fluids 15, 1622 (2003)

5. Y. Ding, N. Gravish, D.I. Goldman, Phys. Rev. Lett. 106, 028001 (2011)

6. F. Guillard, Y. Forterre, O. Pouliquen, Phys. Rev. Lett. 110, 138303 (2013)

7. R. Albert, M.A. Pfeifer, A.L. Barabasi, P. Schiffer, Phys. Rev. Lett. 82, 1, 205-208 (1999)

8. N. Gravish, P.B. Umbanhowar, D.I. Goldman, Phys. Rev. Lett. 105, 128301 (2010)

9. American Association of Cereal Chemists, Official Methods of the AACC, Method 76-13, $10^{\text {th }}$ edition, The Association, St. Paul, MN, USA, (2000)

10. S. Mandato, B. Cuq, T. Ruiz, Eur. Phys. J. E 35, 56 (2012)

11. H.A. Janssen, Zeifschrift verein deutscher ingenieur 39, 1045-1049 (1895).

12. M. Sperl, Granular Matter 8, 59-65 (2006)

13. G. Ovarlez, E. Clément, Eur. Phys. J. E 16, 421-438 (2005)

14. L. Vanel, D. Howell, D. Clark, R.P. Behringer, E. Clément, Phys. Rev. E 60, 5, R5040 (1999)

15. F. Radjai, M. Jean, J.-J. Moreau, S. Roux, Phys. Rev. Lett. 77, 2, 274-277 (1996)

16. G.D.R. MIDI, Eur. Phys. J. E 14, 341-365, (2004) 